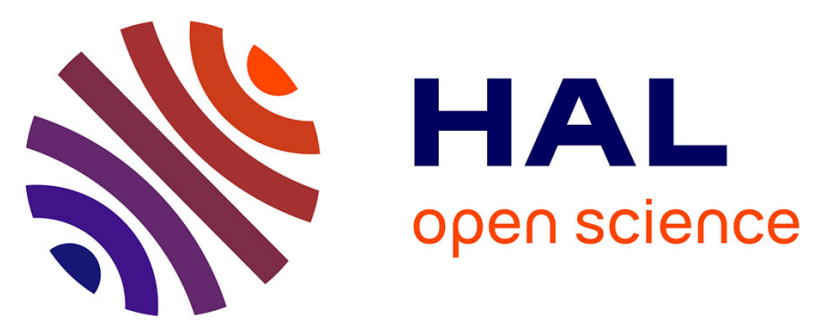

\title{
Assessing the efficiency of industrial exoskeletons with biomechanical modelling -Comparison of experimental and simulation results
}

\author{
Lars Fritzsche, Christian Gärtner, Michael Spitzhirn, Pavel E Galibarov, \\ Michael Damsgaard, Pauline Maurice, Jan Babič
}

\section{To cite this version:}

Lars Fritzsche, Christian Gärtner, Michael Spitzhirn, Pavel E Galibarov, Michael Damsgaard, et al.. Assessing the efficiency of industrial exoskeletons with biomechanical modelling -Comparison of experimental and simulation results. IEA 2021 - 21st Triennal Congress of the International Ergonomics Association, Jun 2021, Vancouver, Canada. pp.353, 10.1007/978-3-030-74614-8_43 . hal-03261786

\section{HAL Id: hal-03261786 \\ https://hal.science/hal-03261786}

Submitted on 16 Jun 2021

HAL is a multi-disciplinary open access archive for the deposit and dissemination of scientific research documents, whether they are published or not. The documents may come from teaching and research institutions in France or abroad, or from public or private research centers.
L'archive ouverte pluridisciplinaire $\mathbf{H A L}$, est destinée au dépôt et à la diffusion de documents scientifiques de niveau recherche, publiés ou non, émanant des établissements d'enseignement et de recherche français ou étrangers, des laboratoires publics ou privés. 


\title{
Assessing the efficiency of industrial exoskeletons with biomechanical modelling - Comparison of experimental and simulation results
}

\author{
Lars Fritzsche ${ }^{1}$, Christian Gärtner ${ }^{1}$, Michael Spitzhirn ${ }^{1}$, Pavel E. Galibarov² ${ }^{2}$, Michael \\ Damsgaard $^{2}$, Pauline Maurice ${ }^{3}$ and Jan Babič ${ }^{4}$ \\ ${ }^{1}$ imk automotive GmbH, Amselgrund 30, 09128 Chemnitz, Germany \\ ${ }^{2}$ AnyBody Technoloy A/S, Niels Jernes Vej 10, 9220 Aalborg, Denmark \\ ${ }^{3}$ Université de Lorraine, CNRS, Inria, F-54000 France \\ ${ }^{4}$ Jožef Stefan Institute, Jamova cesta 39, SI-1000 Ljubljana, Slovenia \\ I ars.fritzsche@mk-aut omt ive.de
}

\begin{abstract}
Exoskeletons are currently introduced for several industrial applications, but in many cases the efficiency of such devices in supporting heavy physical work has not been fully proved yet. Biomechanical simulation could considerably contribute to determining the efficiency of exoskeletons in various use cases with different user populations. In this paper we present an approach to extent laboratory and field studies by using the software AnyBody Modelling System. The biomechanical simulation is applied to the "Paexo Shoulder", a commercial exoskeleton provided by Ottobock. Results show that the exoskeleton substantially reduces muscle activation and joint reaction forces in the shoulder and does not increase activation or forces in the lumbar spine. Comparison with laboratory measurements show very similar results. This indicates that the simulation framework could be used to evaluate changes in internal body loads as a result of wearing exoskeletons and thereby, supplements laboratory experiments and field tests during exoskeleton design and development.
\end{abstract}

Keywords: Musculoskeletal modelling, exoskeleton, biomechanical simulation, industrial exoskeletons, AnyBody Modelling System, Paexo Shoulder.

\section{Introduction}

Industrial exoskeletons can potentially be used for supporting workers in heavy physical tasks that may be associated with high risks for developing musculoskeletal disorders (MSDs). Laboratory experiments and field studies are common approaches to evaluate feasibility and effects of industrial exoskeletons (de Looze et al., 2015). This kind of research is necessary to get valuable and real-world insights into objective measures as well as subjective comfort evaluation and acceptance. However, they often require complex sensor technologies like EMG electrodes that are difficult to 
use in practice. They also do not allow conclusions about internal joint loads or compensatory mechanisms and they are costly or may disrupt ongoing work in field studies. Biomechanical simulations can tackle these challenges because they can be prepared on a computer and safely analyzed using digital human models. They also allow to calculate forces or joint moments inside the body and they can be used for investigating various use cases without expensive experimental set up.

In this paper we present an approach to extent laboratory and field studies by using the biomechanical software AnyBody Modelling System provided by AnyBody Technology A/S, Denmark. The simulation is applied to the "Paexo Shoulder", a commercially available exoskeleton provided by Ottobock SE \& Co. KGaA, Germany. It weighs approx. $1.9 \mathrm{~kg}$ and is specifically designed to support overhead work. Detailed study results are published in Fritzsche et al. (in press).

\section{Methods}

This study applies the simulation framework firstly presented in Galibarov et al. (2019) using a set of data recorded at a laboratory experiment and compares simulation and experimental outcomes. The laboratory experiment included 12 participants (all male) performing an overhead drilling task with a hand-held tool (Figure 1). Detailed descriptions are presented in Maurice et al. (2020), the data set is available on Zenodo (http://doi.org/10.5281/zenodo.1472214). All participants performed the task while wearing the Paexo Shoulder exoskeleton (WE), and without wearing it (NE). Whole body kinematics were retrieved using an Xsens MVN inertial motion tracking suit. Moreover, muscle activities of the right anterior deltoid and right erector spinae longissimus were recorded with Biometrics EMG system. Additionally, heart rate and oxygen consumption were measured as indicators of metabolic effort.

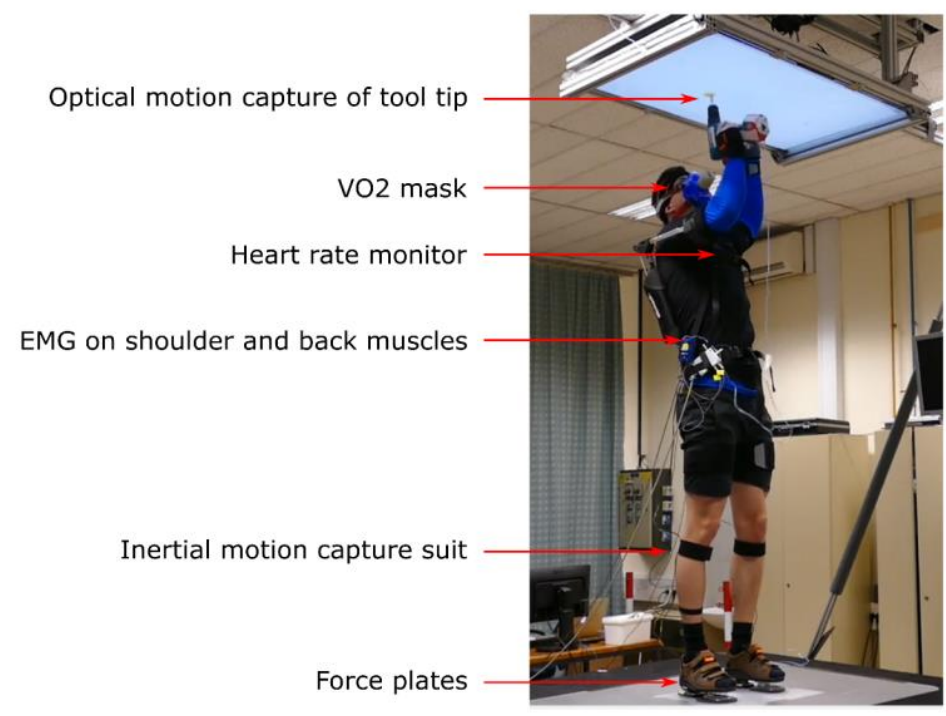

Fig. 1. Experimental set up at the laboratory experiment (adapted from Maurice et al, 2020). 
Using the motion capturing data that were recorded at the laboratory experiment, simulations were created and analyzed with AnyBody Modeling System v.7.3.2 (AMS). AMS uses a biomechanical human model comprised of most of the muscle elements, bones, and joints in the body. The system computes muscle activations, joint moments and reactions forces necessary to generate the specified motions by recruiting muscles in an optimal way (Damsgaard et al., 2006). In this study, a total of 2.880 trials (12 participants, 2 conditions NE/WE, 5 sets of 24 trials) were simulated and processed using a Python script (Lund et al., 2019). The processing model used anthropometric measurements available in the recorded Xsens files to scale corresponding model body parts to consistently represent body size of the participants (Figure 2). Inverse dynamics analysis was then carried out to compute estimations of muscle activities and joint reaction forces.

Based on the repeated-measures design in the laboratory experiment, simulation data was grouped into two conditions "with exoskeleton" (WE) and "without exoskeleton" (NE). Data analysis was also done using a Python script to automatically analyze the 2.880 trials for each simulation variable. Single outlier values beyond three standard deviations above or below the mean were excluded. Descriptive data analysis included calculating box-plots and histograms for each variable. Wilcoxon signed-rank test was used to test for significant differences between the two conditions, since most of the data was not normally distributed according to Kolmogorov-Smirnov-Test. Results were considered as statistically significant with $\alpha<.05$ (two-sided).
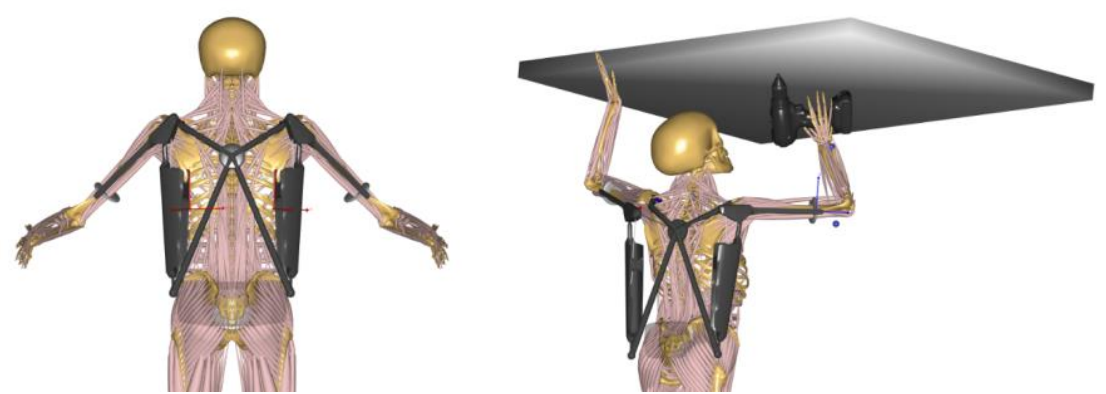

Fig. 2. AnyBody Model with "Paexo Shoulder" exoskeleton (left); application of Paexo Shoulder model in overhead drilling task (right) (adapted from Fritzsche et al., in press).

\section{$3 \quad$ Results}

Results of the AMS biomechanical simulation demonstrate that wearing the exoskeleton reduces muscle activation in the three deltoid shoulder muscles (anterior, posterior, lateral) by $74 \%$ to $87 \%$ compared to the baseline activation with no exoskeleton. Effects were stronger for the right deltoid muscles than for the left deltoid muscles because the drilling tool was used in the right hand, whereas the left hand was only needed to stabilize the body (Figure 1). Similarly, infraspinatus muscle at the shoulder front showed a high baseline activation (NE) at the right side that is reduced by $42 \%$ 
while wearing the exoskeleton (WE). Other relevant muscles in the shoulder/arm area, such as triceps, biceps and trapezius, showed a very low baseline activation $(<10 \%$ of maximal possible activation in both conditions on both sides). This indicates that these muscles were not very much involved in the overhead drilling task, although some of them also showed significant decreases of muscle activation on a low level.

Joint reaction forces in the shoulder were also analyzed by means of AMS simulation. The glenohumeral joint forces were reduced between $56 \%$ and $80 \%$ in all three force directions while wearing the exoskeleton. Similarly, forces in the acromioclavicular joint and in the sternoclavicular joint were reduced between $54 \%$ and $68 \%$ in all directions while wearing the exoskeleton, with one small opposite effect in the sternoclavicular medio-lateral force on a very low force level (4.5 $\mathrm{N}$ increase in WE condition). Overall, results on joint reaction forces are very consistent across different joints and force directions indicating that the exoskeleton is substantially reducing strain in the shoulder joints.

Finally, muscle activities and joint forces in the spine were analyzed with the simulation. Results showed a medium activation pattern in erector spinae muscles on left and right side (approx. $20 \%$ ), but there were no significant differences with or without exoskeleton use. Moreover, compression forces in the L5/S1 disc area were unchanged in two directions or even slightly decreased (approx. $12 \%$ ) while wearing the exoskeleton. Overall, wearing the exoskeleton did not significantly influence muscle activities or joint forces in the lumbar spine.

\section{Discussion}

Results of the AMS simulation study suggest that the Paexo Shoulder exoskeleton is an effective device to reduce biomechanical strain in overhead drilling tasks. Muscle activations of the shoulder complex are reduced, which should decrease the fatigue level of the workers. Similarly, it reduces reaction forces in the shoulder joint supposedly leading to a decrease in shoulder joint cartilage degeneration rates. The device does not redistribute the arm loads onto the lumbar spine, indicating that no adverse side effects for the lumbar spine have to be expected.

Results of the laboratory experiment and the simulation are quite similar for most parameters: (1) in the lab, measured EMG activity in the anterior deltoid muscle is decreased by $54 \%$ in average; in the simulation, the same muscle activity is reduced by $74 \%$ while wearing the exoskeleton. (2) Muscle activity of the erector spinae does not show any difference for with/without exoskeleton conditions in both laboratory measurement and simulation. (3) Metabolic parameters in the lab, such as decreased oxygen consumption $(-33 \%)$ and heart rate $(-19 \%)$, also confirmed that the use of the exoskeleton is related to reduced strain for the entire body.

In summary, these results are suggesting that the simulation framework is a valid approach for investigating the effects of exoskeletons supplementing experimental studies by providing insights into changes inside the human musculoskeletal system. This framework could be extended by analyzing a variety of basic movements/tasks wearing the exoskeleton with different human populations. It also allows investigating 
intended main effects as well as side effects of exoskeletons (and possibly other wearable devices). Such analysis can not only be used for product evaluation, but also for improving the design and functionalities of exoskeletons in the development phase.

Limitations include the exoskeleton fit to the body, because motion of the exoskeleton was not tracked during the experiment (virtual fitting of the exoskeleton was done for each participant according to the manufacturer's guidelines, but may contain some discrepancies with reality). Furthermore, experimental and simulation data can only be compared to some extent, because computed muscle activations represent a percentage of a maximum muscle force needed to perform motions and do not necessarily match EMG signals on an absolute scale.

Future research should take into account that the presented study and the simulation framework was developed based on the evaluation of passive exoskeletons with mechanical components (springs, etc.). It seems more difficult to apply for soft exoskeletons with mainly textile components and active exoskeletons with external power supply. Another field of future research is the question how biomechanical simulations can be prepared without any motion capturing data. AMS already allows to do that, but it still requires to a lot of expertise and effort to create realistic simulations. Other digital human models, such as ema Work Designer (Fritzsche et al., 2019) use algorithms for generating artificial motions with lower effort, which potentially could be used as an input for AnyBody biomechanical simulations in order to make such studies independent from lab recordings and allow evaluation of virtual prototypes.

\section{References}

1. M. Damsgaard, J. Rasmussen, S. Tørholm, E. Surma \& M. de Zee (2006). Analysis of musculoskeletal systems in the AnyBody Modeling System. Sim. Model. Practice and Theory, 14, 1100-1111.

2. M. P. de Looze, T. Bosch, F. Krause, K. S. Stadler \& L. W. O'Sullivan (2016). Exoskeletons for industrial application and their potential effects on physical work load. Ergonomics, 59, 671-681.

3. L. Fritzsche et al. (in press). Assessing the efficiency of exoskeletons in physical strain reduction by biomechanical simulation with AnyBody Modelling System. Wearable Technologies (submitted).

4. L. Fritzsche, S. Ullmann, S. Bauer \& V.J. Sylaja. (2019). Task-based digital human simulation with Editor for Manual Work Activities - Industrial applications in product design and production planning. In G. Paul \& S. Scataglini (Eds.), DHM and Posturography, London, UK: Elsevier. 569-575

5. P. E. Galibarov, M. Damsgaard, M. Spitzhirn, C. Gärtner \& L. Fritzsche (2019). Application of a biomechanical simulation framework to assess effects of an exoskeleton on a human body. Presented at WearRAcon Europe, 2019.

6. M. E. Lund, J. Rasmussen \& M. S. Andersen (2019). AnyPyTools: A Python package for reproducible research with the AnyBody Modeling System. Journal of Open Source Software, 4(33), 1108, https://doi.org/10.21105/joss.01108.

7. P. Maurice et al. (2020). Objective and Subjective Effects of a Passive Exoskeleton on Overhead Work. IEEE Transactions on Neural Systems and Rehabilitation Engineering, $28,152-164$. 